
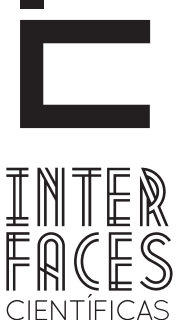

SAÚDE E AMBIENTE

ISSN IMPRESSO 2316-3313

ISSN ELETRÔNICO 2316-3798

DOI - 10.17564/2316-3798.2015v4n1p29-39

\title{
ESTRESSE NA EQUIPE DE ENFERMAGEM: COMO SE MANIFESTA
}

Ana Paula Barros da Silva ${ }^{1}$

Eva Farias de Sousa ${ }^{3}$
Carla Maria Lopes de Vasconcelos Gomes ${ }^{2}$

\section{RESUMO}

Verificar como se manifesta o estresse na equipe de enfermagem; sinais e sintomas estão mais presentes nestes profissionais; e associar o perfil sociodemográfico da população em estudo á manifestação de estresse. Trata-se de um estudo descritivo, quantitativo, amostra composta de Enfermeiros (as), técnicos (as) e auxiliares de Enfermagem, do Hospital Geral do Estado Profo Osvaldo Brandão Vilela ( Maceió-AL), realizado entre 2012 e 2013. Para avaliar os sinais e sintomas de estresse foi utilizando o instrumento validado - Teste de Lipp - ISS - Inventário Sintomas de Stress. população predominantemente feminina (93\%), casada (62\%), idade entre 34 e 41 anos, 55\%, afirmam ter um segundo trabalho e $90 \%$ trabalhar nos turnos (manhã, tarde e noite). Com relação as

sintomas $62 \%$ dos profissionais apresentam sinais e sintomas de estresse, estando $72 \%$ na fase intermediaria, $17 \%$ na fase de contato, e $11 \%$ na fase critica e perigosa. 0 estudo evidenciou que a maioria dos profissionais da equipe de enfermagem apresenta sinais e sintomas de estresse quer seja na exposição intermediaria ou na categoria de alta estresse no trabalho, indicando que requer atenção quanto ao desenvolvimento de ações que minimize os riscos a saúde desses profissionais.

\section{PALAVRAS-CHAVE}

Desgaste profissional. Estresse Ocupacional. Equipe de Enfermagem. Saúde do Trabalhador. 


\section{ABSTRACT}

See how stress manifests in the nursing team; signs and symptoms are more present in these professionals; and associate the sociodemographic profile of the study population will manifestation of stress. This is a descriptive study, quantitative sample of Nurses, technical and auxiliary nursing, the General State Hospital Prof. Osvaldo Brandao Vilela (Maceió-AL), conducted between 2012 and 2013. To assess the signs and symptoms of stress was using the validated instrument - Lipp Test - ISS - Stress Symptoms Inventory. Population predominantly female (93\%), married (62\%), aged between 34 and 41 years, 55\% claim to have a second job and $90 \%$ work in shifts (morning, afternoon and evening). Regarding the symptoms

\section{RESUMEN}

Investigar cómo el estrés se manifiesta en el equipo de enfermería; señales y síntomas están más presentes en estos profesionales; y asociar el perfil sociodemográfico de la población en estudio al manifiesto de estrés. Método: Se trata de un estudio descriptivo cuantitativo, una muestra que constituye de enfermeros(as), técnicos(as) y auxiliares de enfermería, del Hospital Geral do Estado Prof. Osvaldo Brandão Vilela (Maceió-Alagoas), llevado a cabo entre 2012 y 2013. Para evaluar los señales y síntomas de estrés fue utilizado el instrumento validado - Prueba de Lipp - ISS - Inventario de síntomas de estrés. Resultados: población predominantemente femenina (el 93\%), casada (el 62\%), con edades comprendidas entre 34 y 41 años; el 55\% afirman tener un segundo empleo y el $90 \%$ trabajan en los turnos (mañana, tar-
$62 \%$ of professionals have signs and symptoms of stress, with $72 \%$ in the intermediate phase, $17 \%$ in the contact phase, and $11 \%$ in the critical phase and dangerous. The study showed that most nursing team professionals presents signs and symptoms of stress either in the intermediate exposure or in high-stress category at work, indicating that requires attention for the development of actions to minimize the risks to health of these professionals.

\section{KEYWORDS}

Professional Wear Out. Occupational Stress. Nursing Staff. Worker's Health.

de y noche). En relación con los síntomas, el $62 \%$ de los profesionales tienen señales y síntomas de estrés, con un $72 \%$ en la fase intermedia, un $17 \%$ en la fase de contacto, y el $11 \%$ en la fase crítica y peligrosa. Conclusión: El estudio mostró que la mayoría de los miembros de la enfermería presentan señales y síntomas de estrés, sea en la categoría intermedia o en la de alta exposición al estrés en el trabajo, lo que indica que requiere atención al desarrollo de las acciones para minimizar los riesgos a la salud de estos profesionales.

\section{PALABRAS CLAVE}

Desgaste Profesional. Estrés laboral. Equipo de Enfermería. Salud Ocupacional. 


\section{INTRODUÇ̄̃̃O}

Com as mudanças ocorridas no mundo nos últimos tempos, a exigência do mercado de trabalho por profissionais mais especializados é cada vez maior. Todas estas mudanças vêm estabelecendo um estilo de vida às pessoas que acaba alterando suas condições de saúde, causando transtornos psicofisiológicos.

Um destes transtornos é o estresse, ele pode ser resultado de fatores físicos, psicológicos, e sociais. As alterações provocadas por ele é resultado da necessidade do indivíduo de se adaptar a novas situações. 0 estresse é quase sempre visualizado como algo negativo que ocasiona prejuízo no desempenho global do indivíduo (STACCIARINI; TRÓCCOLI, 2001).

Em seus estudos, o médico Canadense Hans Selye (1936), responsável por um dos primeiros estudos sobre o estresse, submeteu cobaias a estímulos estressores e observou um padrão especifico na resposta comportamental e física dos animais. Para este estudioso, o estresse é o estado manifestado por uma síndrome específica, constituído por todas as alterações não-específicas produzidas no sistema biológico (CAMELO; ANGERAM, 2004).

Diante das exigências e desafios enfrentados no dia a dia dos trabalhadores é comum que esses fatores comprometam o estado de saúde de cada um. Nem todos conseguem acompanhar e lidar com essas mudanças e cobranças, além de resultado em tempo hábil, passam a intensificar as jornadas de trabalho, pois não se permitem expressar suas fragilidades. Outros, mesmo sentindo que toda esta carga lhe faz mal, não desistem e muitas vezes ultrapassam daquilo que podem suportar, o que pode contribuir com o adoecimento tanto físico como mental. Para exemplificar o quanto o estresse tem causado mundialmente, a Organização Mundial de Saúde (OMS) afirma que ele já se enquadra como uma "Epidemia global".

0 estresse constitui em um quadro que inclui distorções cognitivas, isto é, um modo inadequado de pensar e avaliar os eventos da vida, vulnerabilidades pessoais e comportamentos observáveis eliciadores. Inclui, também, uma hiper-reatividade fisiológica pe- rante as demandas psicossociais, a qual pode ser gerada por uma hipersensibilidade do sistema límbico, conduzindo a produção excessiva de catecolaminas, testosterona e cortisol (LIPP, 2001).

As investigações têm demonstrado que os eventos estressantes podem vir a serem fatores etiológicos de vários problemas físicos e emocionais. Nessas investigações, o estresse tem sido conceituado, sucessivamente, como estímulo, resposta e interação (LAUTERT; CHAVES; MOURA, 1999).

Os sinais e sintomas que ocorrem com maior frequência de nível físico são: sudorese, tensão muscular, taquicardia, hipertensão, ranger de dentes, hiperatividade, náuseas, mãos e pés frios. Em termos psicológicos, vários sintomas podem ocorrer como: ansiedade, tensão, angústia, insônia, dificuldades interpessoais, dúvidas quanto a si próprio, preocupação excessiva, inabilidade de concentrar-se em outros assuntos que não o relacionado ao estressor, dificuldade de relaxar, ira e hipersensibilidade emotiva (LIPP, 1994).

Tem-se tornado familiar o relato da presença de estresse por profissionais da área da saúde. 0 estresse apresentado por esses profissionais deve vir acompanhado por esforços de enfrentamento para gerenciar as consequências das fontes de estresse e retornar 0 indivíduo a um nível estável de funcionamento homeostático (CAMELO; ANGERAMI, 2004).

A Health Education Authority afirma que a Enfermagem é a quarta profissão mais estressante no setor público, pelo fato de trabalhar com enfermidades críticas e com situações de morte, como acrescentam Murofure, Abranches e Napoleão, 2005. A equipe de enfermagem de uma unidade hospitalar é composta por Enfermeiros, técnicos e auxiliares de enfermagem, que desempenham varias atividades e cuidados destinados a paciente com as mais diversas enfermidades como: administração de medicação, sondagens, punções, curativos, higiene entre outras atividades; além de participar de procedimentos junto à equipe multiprofissional.

Nesse contexto os referidos profissionais se expõem aos mais diversos tipos de riscos, dentre estes 
as situações estressoras, e para poder cuidar da vida de seus pacientes eles precisam estar em boas condições de saúde. 0 seu processo de trabalho, incluindo a estrutura e a organização funcional, sugere que o trabalho da equipe de enfermagem é complexo e de grande responsabilidade. Há um clima de tensão emocional, desgaste físico e psíquico que pode contribuir como fator desencadeante do estresse. Exigindo assim, do profissional, também do profissional enfermeiro uma adaptação em relação a esses agentes estressores para manter o seu equilíbrio homeostático (COSTA; LIMA; ALMEIDA, 2003).

Por razões descritas sobre o estresse no ambiente de trabalho em que as atividades são realizadas sob pressão, exigências físicas e psicológicas, e presença de diversos tipos de riscos. 0 estresse se torna uma constante entre aqueles que realizam esses trabalhos nas instituições de saúde (CASTRO, 2004).

0 interesse no tema em estudo surgiu a partir de experiências vivenciadas pelas pesquisadoras no período do estágio da graduação, que oportunizou observar queixas de dores e irritação entre outras, referidas pelos profissionais. Diante do exposto, surgiu à necessidade de aprofundar as discussões sobre o tema, ao mesmo tempo contribuir para melhoria das condições de trabalho entre estes profissionais. Dessa forma, torna-se relevante responder à pergunta da pesquisa: Quais as principais manifestações de estresse apresentadas pela equipe de enfermagem?

Assim, o objetivo do presente trabalho foi verificar como se manifesta o estresse na equipe de Enfermagem, identificar quais sinais e sintomas estão mais presentes nesses profissionais, e associar o perfil sociodemográfico da população em estudo à manifestação de estresse pela equipe de Enfermagem.

\section{METODOLOGIA}

Trata-se de um estudo de caráter descritivo, com abordagem quantitativa. A pesquisa foi realizada com a equipe de enfermagem (enfermeiros, técnicos e auxiliares de enfermagem) da área azul, que é uma área de grande demanda de pacientes, tanto crianças, quanto adultos, do Hospital Geral do Estado Prof. Osvaldo Brandão Vilela (HGE), localizado em Maceió-AL.

Para participar deste estudo como sujeito foram estabelecidos como critérios de inclusão os profissionais de enfermagem (Enfermeiros (as), Auxiliares e Técnicos (as) em enfermagem) da Área azul do HGE, que estivessem ativos no período da coleta de dados, que tivessem mais de ano de atuação na instituição e que aceitassem participar da pesquisa. E como critérios de exclusão, os integrantes da equipe que trabaIhem a menos de um ano na instituição, os que estiverem afastados do trabalho por férias, licenças medica, licença maternidade ou que se negarem a participar da pesquisa. Dessa maneira, foram respondidos 29 questionários, sendo destes cinco enfermeiros, 14 técnicos e 10 auxiliares de enfermagem.

Esta pesquisa está de acordo com as orientações do Comitê de Ética em Pesquisa Local pertencente à Universidade Estadual de Ciências da Saúde de Alagoas (UNCISAL), por meio do protocolo n $1792 / 12$ e autorização da coordenação do curso de Enfermagem. Todos os participantes foram esclarecidos acerca dos objetivos do estudo e dos procedimentos metodológicos da pesquisa. Portanto, aqueles que concordaram em participar, assinaram um Termo de Consentimento Livre e Esclarecido.

Os dados foram coletados por meio de um instrumento semiestruturado, com questões fechadas que incluiu dados demográficos, funcionais e questões para avaliar os sinais e sintomas de estresse, utilizando o instrumento validado - Teste de Lipp - ISS - Inventário Sintomas de Stress (LIPP, 2005).

Esse instrumento divide os sinais e sintomas em três fases, a primeira é composta por 15 itens que se referem aos sintomas físicos ou psicológicos que o sujeito tenha experimentado nas últimas 24 horas; a segunda fase, composta de dez sintomas físicos e cinco psicológicos, está relacionada aos sintomas no último mês; e a terceira e última fase, composta de 12 sintomas físicos e 11 psicológicos, refere-se a sintomas experimentados nos últimos três meses. Alguns dos sintomas que aparecem na primeira fase voltam a aparecer na terceira fase, mas com intensidade dife- 
rente. No total, o ISS apresenta 37 itens de natureza somática e 19 psicológicas, sendo os sintomas muitas vezes repetidos, diferindo somente em sua intensidade e seriedade. A fase três (quase-exaustão) é diagnosticada na base da frequência dos itens assinalados na fase de resistência.

A cada sete ou mais sintomas marcados nas primeiras 24 horas, o individuo é classificado na fase de contato com a fonte de estresse, com suas sensações típicas na qual o organismo perde o seu equilíbrio e se prepara para enfrentar a situação estabelecida em função de sua adaptação. São sensações desagradáveis, fornecendo condições para reação a estas sendo fundamentais para a sobrevivência do indivíduo (LIPP, 2001).

A ocorrência de quatro ou mais sinais e sintomas é a fase intermediária, em que o organismo procura o retorno ao equilíbrio. Apresenta-se desgastante, com esquecimento, cansaço e duvidosa. Pode ocorrer nesta fase a adaptação ou eliminação dos agentes estressantes e consequente reequilíbrio e harmonia ou evoluir para a próxima fase em consequência da não adaptação e/ou eliminação da fonte de estresse.

Quando ocorrem de nove ou mais sinais e sintomas é dito fase "crítica e perigosa", ocorrendo uma espécie de retorno a primeira fase, porém agravada e com comprometimentos físicos em forma de doenças.

Os 29 questionários utilizados para apresentar os dados desta pesquisa foram obtidos no período de junho de 2012 a setembro de 2013. Pelo fato deste setor ser voltado para assistência de urgências, ocorreu uma grande dificuldade em realizar a coleta de dados para esta pesquisa, pois os profissionais estão constantemente prestando assistência.

A aplicação destes questionários foi realizada de forma padronizada, simultaneamente por duas pesquisadoras, os participantes eram orientados a questionar acerca de dúvidas que viessem a surgir e as aplicadoras dos mesmos permaneciam no local durante todo o tempo.

Após a coleta, inicialmente, realizou-se uma análise exploratória dos dados (Estatística Descritiva). Os cálculos estatísticos foram realizados com o auxílio do Programa EP-INFO.
Os resultados principais foram analisados e, posteriormente, divulgados para os sujeitos da pesquisa por meio de uma revista indexada, em colaboração com o Centro de Estudo da Instituição.

\section{RESULTADOS}

A população alvo foi constituída por 80 trabalhadores de enfermagem (enfermeiros, técnicos e auxiliares de enfermagem) do Hospital Geral do Estado Prof. Osvaldo Brandão Vilela.

Dos sujeitos que se enquadravam no estudo, $63,75 \%$ não participaram da pesquisa por recusa ou por estarem de férias. Restaram 29 trabalhadores que responderam os questionários, correspondendo a uma taxa de resposta de $36,25 \%$.

Para descrever o perfil da amostra em estudo foram elaboradas algumas questões referentes a sexo, idade, estado civil e renda.

Tabela 1 - Perfil dos profissionais de enfermagem da área azul do Hospital Geral do Estado de Alagoas Maceió-AL, 2012-2013

\begin{tabular}{llll}
\hline Variável & Categoria & n & \% \\
\cline { 2 - 4 } & Feminino & 27 & 93 \\
& Masculino & 2 & 7 \\
Sexo & 26 e 33 anos & 4 & 14 \\
Idade & 42 e 41 anos 49 anos & 11 & 38 \\
& 50 anos ou mais & 7 & 24 \\
& Solteiro (a) & 7 & 24 \\
Estado civil & Casado (a) & 3 & 10 \\
& Separado(a)/ & 18 & 62 \\
Renda & Viúvo (a) & 6 & 21 \\
mensal & $<2$ salários & 2 & 7 \\
& $\geq 2$ e $<3$ salários & 5 & 17 \\
& $\geq 3$ e $<5$ salários & 9 & 31 \\
& $\geq 5$ e $<10$ salários & 6 & 21 \\
& $\geq 10$ e $<20$ salários & 1 & 4 \\
\hline
\end{tabular}

Fonte: Dados da pesquisa. 
De acordo com a Tabela 1, a maioria da população te esta entre 34 e 41 anos, 38\% (n: 11), 62\% (n: 18) é do sexo feminino, $93 \%$ ( $n: 27)$, sendo $7 \%$ ( $n: 2)$ do casados. E com relação à renda mensal, 31\% ( profissionais do sexo masculino. A idade predominan- recebem cerca de 2 a 3 salários mínimos.

Tabela 2 - Dados funcionais dos profissionais de enfermagem da área azul do Hospital Geral do Estado de Alagoas - Maceió-AL, 2012-2013

\begin{tabular}{|c|c|c|c|}
\hline Variável & Categoria & $\mathbf{N}$ & $\%$ \\
\hline \multirow{5}{*}{ Cargo } & Enfermeiro & & \\
\hline & & 5 & 17 \\
\hline & Técnico de Enfermagem & 14 & 48 \\
\hline & & 10 & 35 \\
\hline & Auxiliar de Enfermagem & & \\
\hline \multirow{4}{*}{ Tempo na profissão atual } & 3 a 5 anos & 2 & 7 \\
\hline & 5 a 7 anos & 1 & 3 \\
\hline & 7 a 9 anos & 7 & 24 \\
\hline & $>$ de 10 anos & 19 & 66 \\
\hline \multirow{5}{*}{$\begin{array}{l}\text { Tempo de trabalho na } \\
\text { instituição }\end{array}$} & 1 a 3 anos & 5 & 17 \\
\hline & 3 a 5 anos & 4 & 14 \\
\hline & 5 a 7 anos & 2 & 7 \\
\hline & 7 a 9 anos & 9 & 31 \\
\hline & $>$ de 10 anos & 9 & 31 \\
\hline \multirow{3}{*}{ Jornada semanal } & 8 a 12 horas & 1 & 4 \\
\hline & 32 a 40 horas & 14 & 48 \\
\hline & $>40$ horas & 14 & 48 \\
\hline \multirow{3}{*}{ Turno de trabalho } & Manhã e tarde & 2 & 7 \\
\hline & Manhã & 1 & 3 \\
\hline & Todos os horários & 26 & 90 \\
\hline \multirow{6}{*}{ Tem $2^{\circ}$ emprego } & Sim & 16 & 55 \\
\hline & Não & 13 & 45 \\
\hline & 8 a 12 horas & 2 & 12 \\
\hline & 16 a 24 horas & 1 & 6 \\
\hline & 32 a 40 horas & 12 & 76 \\
\hline & $>40$ horas & 1 & 6 \\
\hline
\end{tabular}




$\begin{array}{lllc}\begin{array}{l}\text { Acidente de trabalho no } \\ \text { último ano }\end{array} & \text { Sim } & \mathbf{5} & 17 \\ \begin{array}{l}\text { Noença relacionada ao } \\ \text { trabalho no ultimo ano }\end{array} & \text { Sim } & \mathbf{2 4} & \mathbf{8 3} \\ & \text { Não } & 6 & 21 \\ \text { Usufrui de férias anualmente } & \text { Sim } & \mathbf{2 3} & \mathbf{7 9} \\ & \text { Não } & \mathbf{2 3} & \mathbf{7 9} \\ \text { Satisfação salarial } & \text { Satisfatório } & 6 & 21 \\ & \text { Pouco satisfatório } & 3 & 10 \\ \text { Insatisfatório } & 12 & 42 \\ & & \mathbf{1 4} & \mathbf{4 8}\end{array}$

Fonte: dados da pesquisa.

Com relação aos dados funcionais, na Tabela 2 é indicado que $48 \%$ ( $n: 14)$ da amostra é técnico em enfermagem, $35 \%$ ( $n: 10)$ são auxiliares de enfermagem, e 17\% (n: 5) são enfermeiros (as). 0 tempo de profissão destes profissionais está superior a 10 anos, 66\% (n: 19), já o tempo de trabalho nesta instituição prevalecem os que trabalham de sete a nove anos, $31 \%$ (n: 9), e com a mesma porcentagem, os a mais de 10 anos, $31 \%$ (n: 9 ). 0 tempo de trabalho destes profissionais neste setor é prevalente entre três a cinco anos, 32\% (n: 9).

A jornada de trabalho desses profissionais esta prevalente entre 32 a 40 horas semanais, $48 \%$ (n: 14), e esta mesma porcentagem está para os que trabalham mais de 40 horas por semana. Os turnos de trabalho são distribuídos em todos os horários, matutino, vespertino e também noturno, somando $90 \%$ (26), indicando que os profissionais são plantonistas. $55 \%$ (n: 16) afirmam ter um segundo trabalho, aonde sua jornada semanal de trabalho vai de 32 a 40 horas, $76 \%$ (n: 12).

Quando questionado se o profissional já sofreu algum acidente de trabalho no último ano, $83 \%$ (n: 24) afirmam que não e $17 \%$ (n: 5) afirmam que sim. Se no último ano apresentaram alguma doença relacionada ao trabalho, 79\% (n: 23) afirmam que não. Também foi questionada a situação do trabalhado em relação a férias anuais, $79 \%$ (n: 23) afirmam que usufruem de férias anualmente. Já em relação à satisfação salarial, $48 \%$ ( $n: 14$ ) afirmam estarem insatisfeitos com o salário que recebem.

Tabela 3 - Correlação entre sinais e sintomas e as fases do estresse em profissionais de enfermagem da área azul do Hospital Geral do Estado de Alagoas Maceió-AL, 2012-2013

\begin{tabular}{|c|c|c|c|}
\hline Variável & Categoria & $\mathbf{n}$ & $\%$ \\
\hline \multirow{2}{*}{$\begin{array}{l}\text { Presença se sinais e } \\
\text { sintomas de estresse }\end{array}$} & Sim & 18 & 62 \\
\hline & Não & 11 & 38 \\
\hline \multirow{3}{*}{ Fase de estresse } & I - de contato & 3 & 17 \\
\hline & II- intermediaria & 13 & 72 \\
\hline & $\begin{array}{l}\text { II- critica e } \\
\text { periaosa }\end{array}$ & 2 & 11 \\
\hline
\end{tabular}

A última etapa do levantamento de dados é com relação se o profissional apresenta sinais e sintomas de estresse, e se eles apresentem qual fase de estresse está. Segundo a Tabela 3, 62\% (n: 18) dos profissionais apresentam sinais e sintomas de estresse, estando $72 \%$ ( $n: 13$ ) na fase intermediaria, $17 \%(n: 3)$ na fase de contato, e $11 \%(n: 2)$ na fase critica e perigosa. 
Tabela 4 - Sinais e sintomas, segundo as fases de estresses, encontrados em profissionais da equipe de enfermagem da área azul do Hospital Geral do Estado de Alagoas - Maceió-AL, 2012-2013

\begin{tabular}{lll}
\hline Variável & Categoria & $\mathbf{\%}$ \\
\hline Fase de contato & Vontade de novos projetos & 15 \\
& Mudança de apetite & 15 \\
& Insônia & 15 \\
& Diarreia & 15 \\
& Tensão muscular & 10 \\
& Dor de estômago & 10 \\
Fase & Cansaço & 10 \\
intermediária & & \\
& Desgaste físico & 14 \\
& Problemas de memória & 12 \\
& Irritabilidade & 8 \\
Fase crítica & Diminuição do libido & 8 \\
& Diarreias & 7 \\
& Tonturas & 7 \\
& Cansaço & 7 \\
& Pensamentos repetitivos & 7 \\
& Depressão & 7 \\
& Pesadelos & 7 \\
& Úlceras & 4 \\
& Ansiedade & 4 \\
\hline
\end{tabular}

Fonte: Dados da pesquisa.

Segundo a Tabela 4, os sinais e sintomas mais apresentados durante a fase de contato são: vontade de novos projetos (15\%), mudança de apetite (15\%), insônia (15\%), diarreia (15\%), tensão muscular (10\%) e dor no estomago (10\%). Já na fase intermediária, os sinais e sintomas mais aparentes são: cansaço (10\%), desgaste físico (14\%), problemas de memória (12\%), irritabilidade (8\%) e diminuição do libido (8\%). Na fase crítica os sinais e sintomas mais apresentados são: diarreias (7\%), tonturas (7\%), cansaço (7\%), pensamentos repetitivos (7\%), depressão (7\%), pesadelos (7\%), úlceras (4\%) e ansiedade (4\%).

\section{DISCUSSÃO}

O primeiro objetivo desse estudo é saber como o profissional de Enfermagem manifesta o estresse, porém, antes disso, é preciso saber a porcentagem de in- divíduos que se enquadram em algumas das fases de estresse. De acordo com os dados levantados, $62 \%$ da amostra se encaixam em alguma das fases de estresse.

Os dados encontrados no estudo, $72 \%$ dos componentes da equipe de enfermagem se encontram na fase intermediária do estresse, que é a fase que o organismo procura o retorno ao equilíbrio. Os sinais e sintomas que mais foram relatados pelos profissionais são. Cansaço (10\%), desgaste físico (14\%), problemas de memória (12\%), irritabilidade (8\%) e diminuição do libido (8\%), sem a maioria desse sinais e sintomas psicológicos.

Em seus estudos, Salvador, Silva e Lisboa (2013), cotidianamente os indivíduos estão expostos ao estresse mental ou psicológico, e a capacidade de reagir a isso é uma resposta natural e que se faz necessária. Porém, uma reação exacerbada ao estresse psicológico os torna mais suscetíveis ao desenvolvimento da hipertensão arterial, a qual pode provocar problemas cardiovasculares e/ou morte. Os casos de falha da memória estão relacionados ao cansaço, às poucas horas de sono, a muito trabalho e ao estresse.

Pesquisa realizada com profissionais de um pronto atendimento relatou que estes profissionais percebem mais os sintomas físicos do estresse que os psíquicos, associados à resistência dos profissionais, provocando negação dos sintomas psíquicos. Este achado contradiz o presente estudo, em que foi observado maior destaque aos sintomas psicológicos do que físicos (SELEGHIM ET AL., 2012).

O desgaste mental é encontrado em profissões como a enfermagem, cujo contato pessoal exige dedicação excessiva, carga de trabalho exaustiva, com potenciais conflitos com clientes, chefias e colegas de trabalho (COELHO; ARAUJO, 2010).

Outros sinais e sintomas como insônia (15\%), mudança de apetite (15\%), dores de estomago (10\%), aparecem na fase de contato, que se dá no momento em que o paciente começa a ter, como o nome sugere, contato com as fontes causadores de estresse. Segundo o estudo de Coelho e Araujo (2010), as colocações da equipe evidenciam a fadiga mental, que não pode ser dissociada da fadiga física. A fadiga acumulada 
afeta o organismo, causando insônia, irritabilidade, desânimo, dores e perda de apetite, dentre outros sintomas. Destacamos que o desgaste físico e mental não deve ser menosprezado ou ignorado, pois pode gerar consequências para a saúde mental da equipe de enfermagem e para o cuidado prestado.

Na maioria dos hospitais o trabalho da enfermagem tem sido apontado como bastante estressante. 0 estresse do enfermeiro pode se justificar pela grande responsabilidade e pela baixa autonomia, as quais refletem situações com vários pontos de tensão, determinantes do estresse. 0 nível de pressão exercido pela organização do trabalho, a exigência de maior produtividade, associada à redução contínua do contingente de trabalhadores, à pressão do tempo e ao aumento da complexidade das tarefas, além de expectativas irrealizáveis e as relações de trabalho tensas e precárias, podem gerar tensão, fadiga e esgotamento profissional, constituindo-se em fatores responsáveis por situações de estresse relacionado com o trabalho (SCHMIDT ET AL., 2009).

No estudo de Salomé, Martins e Espósito (2009), os profissionais citam em suas falas que convivem com sentimentos díspares: o cansaço, o esgotamento, a angústia, impotência e dores devido à sobrecarga de trabalho, provocada pela falta de material e de profissionais. Mesmo diante destas circunstâncias, os profissionais procuram prestar uma assistência de qualidade. Tudo isso é observado no presente estudo, e o cansaço aparece tanto em profissionais que estão na fase intermediária, quanto os que estão na fase crítica, $10 \%$ e $7 \%$, respectivamente.

A literatura aponta que ainda há divergências em relação às possíveis causas do estresse ocupacional (COSTA; MARTINS, 2011). Ainda assim, acredita-se que, quanto mais este assunto for estudado, mais contribuições para o conhecimento sobre estresse ocupacional de profissionais de enfermagem surgirá.

Outro objetivo deste estudo é associar o perfil sociodemográfico da população em estudo à manifestação de estresse pela equipe de Enfermagem.

Para Seleghim e outros autores (2012) a enfermagem, além do desenvolvimento de atividades com pacientes críticos e em risco eminente de morte, as características pessoais das trabalhadoras apresentaram vários fatores considerados de risco para 0 desenvolvimento de estresse, como o sexo feminino, ciclo vital adulto, estado civil casado, ter filhos, duplo vínculo e trabalho no período noturno. Todas essas características são encontradas na amostra da pesquisa, onde $93 \%$ da população são do sexo feminino, $38 \%$ têm entre 34 e 41 anos de idade, 62\% são casadas, 55\% têm um segundo trabalho e $90 \%$ trabalham todos os turnos (manhã, tarde e noite).

Já no estudo de Costa e Martins (2011), é identificado que profissionais mais jovens sentiam maior nível de estresse no trabalho. Os autores não apresentam explicação para os resultados obtidos. No entanto, pela própria literatura pesquisada, faz-se uma sugestão de explicação, que deve ser investigada em futuros estudos. Uma situação, para ser percebida como estressora, depende da avaliação cognitiva e da capacidade de enfrentamento do indivíduo. Assim, acredita-se que possa haver alguma relação entre maturidade e melhores esforços e estratégias de enfrentamento e, portanto, menos níveis de estresse. Esta é uma hipótese explicativa porque este estudo não investigou estratégias de enfrentamento, mas uma possibilidade que deve ser investigada em futuras pesquisas.

Contudo, o estresse em mulheres ocorre com maior frequência pela sobrecarga de tarefas características do mundo feminino, pela dupla ou tripla jornada de trabalho. Fator "sexo feminino" alia-se à idade adulta, com responsabilidades e exigências geradas por esta fase da vida, como a necessidade de autorrealização pessoal e profissional e o provimento de recursos financeiros para o sustento da família, bem como ao estado civil e à presença e responsabilidade com filhos como indicadores de sobrecarga, pelo acúmulo das funções intrafamiliares (SELEGHIM ET AL., 2012).

0 tipo de instituição em que atuam os profissionais de enfermagem, categoria profissional, jornada semanal de trabalho e a presença de duplo vínculo empregatício são fatores importantes na vida dos trabalhadores, podendo estar integrados à percepção do estresse ocupacional e por isso foram incluídos neste estudo. 


\section{CONCLUSÃO}

A partir dos achados, o presente estudo refuta a hipótese defendida, a priori, de ocorrência do estresse nos enfermeiros pesquisados. Em síntese, o estresse mostrou ser relativo a situações em que o profissional possa estar inserido, e ao seu perfil.

A população do estudo apontou ser predominantemente feminina (93\%), casada (62\%), idade entre 34 e 41 anos, 55\% afirmam ter um segundo trabalho e $(90 \%)$ trabalhar nos turnos (manhã, tarde e noite). Com relação aos sintomas $62 \%$ dos profissionais apresentam sinais e sintomas de estresse, estando $72 \%$ na fase intermediária, $17 \%$ na fase de contato, e $11 \%$ na fase crítica e perigosa.

A presença de estresse e a dificuldade de enfrentá-lo podem ocasionar alterações físicas e mentais. 0 indivíduo com estresse ocupacional pode levar seus problemas para o ambiente de trabalho, para a vida familiar e vice-versa, inibindo seu convívio em família, podendo gerar, também, um déficit nas áreas afetiva e social. Sendo assim, o estresse no trabalho pode afetar várias áreas da vida do indivíduo, dificultando a manutenção de sua qualidade de vida, indicando que requer atenção quanto ao desenvolvimento de ações que minimize os riscos a saúde desses profissionais.

\section{REFERÊNCIAS}

CAMELO, S. H. H.; ANGERAM, E. L. S. Sintomas de estresse nos trabalhadores atuantes em cinco núcleos de saúde da família. Rev Latino-am Enfermagem, v.12, n.1, 2004. p.14-21.

\section{CASTRO, M. E. S. Condições de trabalho e fatores de risco à saúde dos trabalhadores de Central de material e esterilização do hospital das clinicas de UFP. 2004.}

COELHO, J. A. B.; ARAUJO, S. T. C. Desgaste da equipe de enfermagem no centro de tratamento de queimados. Acta Paul Enferm, v.23, n.1, 2010. p.60-64.
COSTA, D. T.; MARTINS, M. C. F. Estresse em profissionais de enfermagem: impacto do conflito no grupo e do poder do médico. Rev Esc Enferm USP, v.45, n.5, 2011. p.1191-1198.

COSTA, J. R. A.; LIMA, J. V.; ALMEIDA, P. C. Stress no trabalho do enfermeiro. Rev Esc Enferm USP, v.37, n.3, 2003. p.63-71.

LAUTERT, L.; CHAVES, E. H. B.; MOURA, G. M. S. S. $O$ estresse na atividade gerencial do enfermeiro.

Rev Panam Salud Publica/Pan Am J Public Health, v.6, n.6, 1999.

LIPP, M. E. N. Estresse emocional: a contribuição de estressores internos e externos. Rev. Psiq. Clín., 2001.

LIPP, M. E. N. Manual do inventário de sintomas de stress para adultos de Lipp. 3.ed. Recife: Casa do Psicólogo, 2005.

\section{LIPP, M. E. N.; ROCHA, J. C. Stress, hipertensão} arterial e qualidade de vida. São Paulo: Papirus, 1994.

MUROFURE, N. T.; ABRANCHES, S. S.; NAPOLEÃO, A. A. Reflexões sobre estresse e Burnout e a relação com a enfermagem. Rev. Latino-Am. Enfermagem, v.13, n.2, 2005. p.255-261.

SALOMÉ, G. M.; MARTINS, M. F. M. S.; ESPÓSITO, V. H. C. Sentimentos vivenciados pelos profissionais de enfermagem que atuam em unidade de emergência. Rev Bras Enferm, Brasília, v.62, n.6, p.856-62, 2009.

SALVADOR, R. S. P.; SILVA, B. A. S. A.; LISBOA, M. T. L. Estresse da equipe de enfermagem do corpo de bombeiros no atendimento pré-hospitalar móvel.

Esc Anna Nery, v.17, n.2, 2013. p.361-368.

SCHMIDT, D. R. C.; DANTAS, R . A. S.; MARZIALE, M. H. P.; LAUS, A. M. Estresse ocupacional entre profis- 
sionais de enfermagem do bloco cirúrgico. Texto

Contexto Enferm, Florianópolis, v.18, n.2, 2009. p.330-733.

SELEGHIM, M. R et al. Sintomas de estresse em trabalhadoras de enfermagem de uma unidade de pronto socorro. Rev Gaúcha Enferm, v.33, n.3, 2012. p.165-173.
STACCIARINI J.M.R.; TRÓCCOLI, B.T. O estresse na atividade ocupacional do enfermeiro. Rev Latino-am Enfermagem, v.9, n.2, 2001. p.17-25. 